This item was submitted to Loughborough's Research Repository by the author.

Items in Figshare are protected by copyright, with all rights reserved, unless otherwise indicated.

\title{
A dynamical theory of spike train transitions in networks of integrate-and-fire
} oscillators

PLEASE CITE THE PUBLISHED VERSION

LICENCE

CC BY-NC-ND 4.0

REPOSITORY RECORD

Bressloff, P.C., and S. Coombes. 2019. "A Dynamical Theory of Spike Train Transitions in Networks of Integrate-and-fire Oscillators". figshare. https://hdl.handle.net/2134/824. 


\title{
A DYNAMICAL THEORY OF SPIKE TRAIN TRANSITIONS IN NETWORKS OF INTEGRATE-AND-FIRE OSCILLATORS*
}

\author{
P. C. BRESSLOFF ${ }^{\dagger}$ AND S. COOMBES $\ddagger$
}

\begin{abstract}
A dynamical theory of spike train transitions in networks of pulse-coupled integrateand-fire (IF) neural oscillators is presented. We begin by deriving conditions for 1:1 frequency locking in a network with non-instantaneous synaptic interactions. This leads to a set of phase equations determining the relative firing times of the oscillators and the self-consistent collective period. We then investigate the stability of phase-locked solutions by constructing a linearized map of the firing times and analyzing its spectrum. We establish that previous results concerning the stability properties of IF oscillator networks are incomplete since they only take into account the effects of weak coupling instabilities. We show how strong coupling instabilities can induce transitions to non-phase locked states characterized by periodic or quasiperiodic variations of the inter-spike intervals on attracting invariant circles. The resulting spatio-temporal pattern of network activity is compatible with the behavior of a corresponding firing rate (analog) model in the limit of slow synaptic interactions.
\end{abstract}

Key words. pulse-coupled oscillators, integrate-and-fire, quasiperiodicity, Hopf bifurcations

AMS subject classifications. 34,92

1. Introduction. The dynamics of networks of biological neural oscillators continues to be a subject of both theoretical and experimental interest. A major motivation for such interest has been the recent observation that synchronization and waves of excitation can occur during sensory processing [20,36]. Oscillations are also found in the reticular thalamic nucleus (RTN), which is thought to act as a pacemaker for synchronous spindle oscillations observed during sleep or anesthesia [33, 18]. Another important example arises in central pattern generators underlying locomotion [25]. Modeling the biophysical mechanisms underlying neural oscillations requires a system of smooth differential equations with several degrees of freedom for each oscillator as exemplified by the Hodgkin-Huxley model of excitable nerve tissue. This describes the generation of an action potential due to the activation-deactivation of a number of voltage-dependent ionic gates [22]. Analyzing a network of such neurons is a difficult task due to the complexity of the single-neuron model. This motivates the application of phase reduction methods (valid for weak coupling) to reduce the network dynamics to a system in which the relative phase between oscillators is the relevant dynamical variable [14, 21, 29]. However, it has not yet been established that weak coupling between nerve cells is a justifiable assumption.

An alternative approach to studying coupled neural oscillators is to neglect details concerning the shape of a pulse by considering an integrate-and-fire (IF) oscillator model [30] or the related spike response model [16]. The state of an integrate-and-fire oscillator changes discontinuously (resets) whenever it crosses some threshold so that a complete description in terms of smooth differential equations is no longer possible. Mirollo and Strogatz [26] have proven rigorously that globally coupled IF oscillators almost always synchronize in the presence of instantaneous excitatory interactions.

\footnotetext{
*Submitted to SIAM J. Appl. Math. This research was supported by grant number GR/K86220 from the EPSRC (UK).

${ }^{\dagger}$ Nonlinear and Complex Systems Group, Department of Mathematical Sciences, Loughborough University, Loughborough, Leicestershire, LE11 3TU, U. K. P.C.Bressloff@Lboro.ac.uk

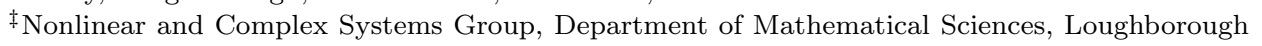
University, Loughborough, Leicestershire, LE11 3TU, U. K. S.Coombes@Lboro.ac.uk
} 
Subsequently, this result has been extended to take into account the effects of synaptic processing and axonal transmission delays $[10,11,15,21,32]$. In particular, one finds that inhibitory (excitatory) synapses synchronize if the rise time of a synapse is longer (shorter) than the duration of an action potential, whereas slow excitatory synapses tend to generate anti-synchrony. Analogous results have been obtained in the spike response model for large networks using mean-field techniques [16, 17]. Traveling waves of synchronized activity have also been investigated, both in finite chains of IF oscillators (modeling locomotion in simple vertebrates) [7, 9], and in two-dimensional networks where spirals and target patterns are observed [24].

One of the interesting features of IF networks is that phase-locked solutions, such as synchronous and traveling wave states, can be described in terms of a set of phase equations involving the relative shifts in the firing times of the oscillators (with the collective frequency of the oscillators determined self-consistently). These steady-state phase equations have the same formal structure as those obtained using phase reduction methods but do not require any restrictions on the strength of the coupling $[8,10]$. This raises the important question as to whether or not the full IF model exhibits new dynamical phenomena for moderate or strong coupling that are not found in the corresponding phase reduced models. Resolving this issue is the main subject of our paper.

We begin by deriving general conditions for phase-locking in networks of IF oscillators with synaptic interactions. We then determine the linear stability of these phase-locked solutions by constructing a linearized map of the oscillator firing times under perturbations of a given phase-locked state (see $\S 2$ and $\S 4$ ). Analysis of the linearized map's spectrum generates conditions for the onset of a discrete Hopf bifurcation in the firing times as the strength of coupling is increased. This identifies regions in parameter space where there are transitions to states with inhomogeneous and (quasi)periodic variations in the interspike intervals (ISIs) on attracting invariant circles. We relate the occurrence of strong coupling instabilities in IF networks to corresponding instabilities in a rate model, for which the output of a neuron is represented by a short-term firing rate (see $\S 3$ ). Strong instabilities of the rate model involve the destabilization of a homogeneous low activity state. This can occur either via a static bifurcation leading to the formation of an inhomogeneous stationary activity pattern (oscillator death) or via a Hopf bifurcation resulting in time-periodic variations in the firing rate (bursting). We establish through numerical examples in $\S 4$ and $\S 5$ how, in the case of slow synapses, there is good agreement between the two models on an appropriately defined time-scale. First, a globally coupled network with inhibitory coupling is shown to desynchronize (in the sense that the in-phase state becomes unstable) at some critical coupling leading to deactivation of some of the oscillators in the network (oscillator death). Such behavior is contrasted with a weak coupling instability in a corresponding excitatory network. Interestingly it is found that the strong coupling instability only occurs if the rate of synaptic response, as characterized by an inverse rise-time $\alpha$, is sufficiently slow. In other words, there exists a critical inverse rise-time $\alpha_{0}(N)$, where $N$ is the number of oscillators, such that the network remains synchronized for arbitrary coupling when $\alpha>\alpha_{0}(N)$. Moreover, $\alpha_{0}(N)$ is a monotonically decreasing function of $N$. This implies that there is a greater tendency for coherent oscillations in large networks, which is consistent with the mode-locking theorem of Gerstner et al [17]. Finally in $\S 5$ we show how a ring network with unidirectional coupling desynchronizes via a strong instability leading either to oscillator death (even $N$ ) or bursting (odd $N$ ). In the latter case the ISIs of 
the oscillators are found to lie on closed periodic orbits. Again such instabilities are only found to occur for slow synapses.

2. Phase-locking in networks of integrate-and-fire oscillators. Consider a network of $N$ integrate-and-fire (IF) neural oscillators. Let $U_{i}(t)$ denote the state of the $i$ th oscillator at time $t, i=1, \ldots, N$. Suppose that the variables $U_{i}(t)$ evolve according to the set of equations

$$
\frac{\mathrm{d} U_{i}(t)}{\mathrm{d} t}=-U_{i}(t)+I_{i}+g \sum_{j=1}^{N} W_{i j} \int_{0}^{\infty} J(\tau) E_{j}(t-\tau) d \tau
$$

supplemented by the reset conditions

$$
U_{i}\left(t^{+}\right)=0 \quad \text { whenever } \quad U_{i}(t)=1, \quad i=1, \ldots, N .
$$

Here $E_{j}(t)$ represents the sequence of pulses transmitted from the $j$ th oscillator, $J(\tau)$ is a distribution of delays normalized such that $\int_{0}^{\infty} J(\tau) d \tau=1$, and $I_{i}$ is a timeindependent external input. The effective strength of the synaptic coupling from the $j$ th oscillator to the $i$ th oscillator is given by $g W_{i j}$ with the parameter $|g|$ controlling the overall strength of the interactions. Time is measured in units of the decay constant of the oscillators, which we take to be of the order $10 \mathrm{~ms}$. Neglecting the shape of an individual pulse, the output spike train of each oscillator is represented as a sequence of Dirac delta-functions,

$$
E_{i}(t)=\sum_{m=-\infty}^{\infty} \delta\left(t-T_{i}^{m}\right)
$$

where $T_{i}^{m}$ are the firing times of the $i$ th oscillator, that is, $U_{i}\left(T_{i}^{m}\right)=1$. Each spike is converted to a post-synaptic potential whose shape is specified by the distribution $J(\tau)$. A biologically motivated choice for $J(\tau)$ is the so-called $\alpha$-function [23]

$$
J(\tau)=\alpha^{2} \tau \mathrm{e}^{-\alpha \tau} \Theta(\tau)
$$

where $\Theta$ is the unit step-function, $\Theta(\tau)=1$ if $\tau>0$ and zero otherwise. This ensures that the maximum synaptic response occurs at a non-zero delay $\tau=\alpha^{-1}$. It is a simple matter to incorporate into the definition of $J(\tau)$ other features such as axonal transmission delays [11] and dendritic delays [4], although we shall not do so here. (Note that considerable analytical simplification is obtained by treating the synaptic interactions of equation (2.1) in terms of direct current injections rather than changes in conductances. We expect the basic results of our paper to carry over to these more biologically realistic forms of interaction).

2.1. Existence of phase-locked states. Following Ref. [7, 8, 9, 10, 32], we restrict our attention to phase-locked solutions of equations (2.1) and (2.2) in which every oscillator resets or fires with the same period $T$. This period must be determined self-consistently. The state of each oscillator is then characterized by a constant phase $\phi_{i} \in \mathbb{R} \backslash \mathbb{Z}$ such that the firing times are of the form $T_{i}^{m}=\left(m-\phi_{i}\right) T$ for all $m \in \mathbb{Z}$ and $i=1, \ldots, N$. For a given set of phases $\Phi=\left(\phi_{1}, \ldots, \phi_{N}\right)$, we integrate equation (2.1) over the interval $t \in\left(-T \phi_{i}, T-T \phi_{i}\right)$ and incorporate the reset condition (2.2) by setting $U_{i}\left(-\phi_{i} T\right)=0$ and $U_{i}\left(T-\phi_{i} T\right)=1$. This leads to the set of $N$ equations

$$
1=\left(1-\mathrm{e}^{-T}\right) I_{i}+g \sum_{j=1}^{N} W_{i j} K\left(\phi_{j}-\phi_{i}, T\right)
$$


where

$$
K(\phi, T)=\mathrm{e}^{-T} \int_{0}^{T} \mathrm{e}^{\tau} J(\tau / T+\phi, T) \mathrm{d} \tau
$$

and

$$
J(\phi, T)=\sum_{m \in \mathbb{Z}} J([\phi+m] T)
$$

Both $J(\phi, T)$ and $K(\phi, T)$ are periodic functions of $\phi$ with unit period. After choosing some reference oscillator, equations (2.5) determine $N-1$ relative phases and the collective period $T$.

In the case of the $\alpha$-function (2.4), both $J(\phi, T)$ and the phase-interaction function $K(\phi, T)$ can be calculated explicitly. First,

$$
J(\phi, T)=\frac{\alpha^{2} \mathrm{e}^{-\alpha T \phi}}{1-\mathrm{e}^{-\alpha T}}\left[T \phi+\frac{T \mathrm{e}^{-\alpha T}}{\left(1-\mathrm{e}^{-\alpha T}\right)}\right], 0 \leq \phi<1 .
$$

Second, decomposing equation (2.6) as

$$
\begin{aligned}
K(\phi, T)= & \int_{0}^{T(1-\phi)} \mathrm{e}^{\tau-T} J(\tau / T+\phi, T) \mathrm{d} \tau \\
& +\int_{T(1-\phi)}^{T} \mathrm{e}^{\tau-T} J(\tau / T+\phi-1, T) \mathrm{d} \tau
\end{aligned}
$$

and using equation (2.8) leads to the result (for $0 \leq \phi<1$ )

$$
\begin{gathered}
K(\phi, T)=\frac{\alpha^{2}}{1-\alpha} \frac{1-\mathrm{e}^{-T}}{1-\mathrm{e}^{-\alpha T}}\left[K_{1}(T) \mathrm{e}^{-\alpha T \phi}+T \phi \mathrm{e}^{-\alpha T \phi}+K_{2}(T) \mathrm{e}^{-T \phi}\right] \\
K_{1}(T)=\frac{T \mathrm{e}^{-\alpha T}}{1-\mathrm{e}^{-\alpha T}}-\frac{1}{1-\alpha}, \quad K_{2}(T)=\frac{1}{1-\alpha} \frac{1-\mathrm{e}^{-\alpha T}}{1-\mathrm{e}^{-T}}
\end{gathered}
$$

For general delay kernels $J(\tau)$ it is more useful for numerical calculations to consider the following Fourier series representation for $K(\phi, T)$ :

$$
K(\phi, T)=\frac{1-\mathrm{e}^{-T}}{T} \sum_{m \in \mathbb{Z}} \frac{\widetilde{J}\left(\omega_{m}\right) \mathrm{e}^{2 \pi i m \phi}}{1+i \omega_{m}}, \quad \omega_{m}=\frac{2 \pi m}{T},
$$

which is convergent provided that $\widetilde{J}(\omega) \rightarrow 0$ as $\omega \rightarrow \pm \infty$. In the particular case of the $\alpha$-function,

$$
\widetilde{J}(\omega) \equiv \int_{-\infty}^{\infty} \mathrm{e}^{-i \omega \tau} J(\tau) d \tau=\frac{\alpha^{2}}{(\alpha+i \omega)^{2}}
$$

In previous work we have analyzed phase-locked solutions of equations (2.5) for a number of distinct types of network. First, in the case of a ring of identical IF oscillators with symmetric coupling we have used group theoretic methods to classify all phase-locked solutions, and have constructed bifurcation diagrams showing how new solution branches emerge via spontaneous symmetry breaking $[8,10]$. Second, in the case of a finite chain of IF oscillators with a gradient of external inputs and anisotropic nearest-neighbor coupling, we have proven the existence of "traveling wave" solutions in which the phase varies monotonically along the chain (except in some narrow boundary layer); such systems are used to model locomotion in simple vertebrates $[7,9]$. 
2.2. Stability of phase-locked states. The stability of phase-locked solutions of an IF network can be analyzed by considering perturbations of the firing times $[31,5,6]$

$$
T_{i}^{n}=\left(n-\phi_{i}\right) T+u_{i}^{n}
$$

First, integrate equation (2.1) from $T_{i}^{n}$ to $T_{i}^{n+1}$ to generate the nonlinear firing time map

$$
\mathrm{e}^{T_{i}^{n+1}}=I_{i}\left[\mathrm{e}^{T_{i}^{n+1}}-\mathrm{e}^{T_{i}^{n}}\right]+g \sum_{j=1}^{N} W_{i j} \sum_{m \in \mathbb{Z}} \int_{T_{i}^{n}}^{T_{i}^{n+1}} \mathrm{e}^{t} J\left(t-T_{j}^{m}\right) d t
$$

Substitute equation (2.14) into equation (2.15) and expand as a power series in the perturbations $u_{i}^{n}$. To $\mathcal{O}(1)$ we recover the phase-locking equations (2.5), whereas the $\mathcal{O}(u)$ terms lead to an infinite-order linear difference equation given by

$$
A_{i}(\Phi, T)\left[u_{i}^{n+1}-u_{i}^{n}\right]=g \sum_{j=1}^{N} W_{i j} \sum_{m \in \mathbb{Z}} G_{m}\left(\phi_{j}-\phi_{i}, T\right)\left[u_{j}^{n-m}-u_{i}^{n}\right]
$$

where

$$
\begin{gathered}
A_{i}(\Phi, T)=I_{i}-1+g \sum_{j=1}^{N} W_{i j} J\left(\phi_{j}-\phi_{i}, T\right) \\
G_{m}(\phi, T)=\int_{0}^{T} \mathrm{e}^{t-T} J^{\prime}(t+(m+\phi) T) d t
\end{gathered}
$$

and $J^{\prime} \equiv d J / d t$.

Equation (2.16) has a discrete spectrum that can be found by taking $u_{j}^{n}=\mathrm{e}^{n \lambda} v_{j}$ with $\lambda \in \mathbb{C}$ and $0 \leq \operatorname{Im}(\lambda)<2 \pi$. This leads to the eigenvalue equation

$$
A_{i}(\Phi, T)\left(\mathrm{e}^{\lambda}-1\right) v_{i}=g \sum_{j=1}^{N} W_{i j}\left[\widetilde{G}\left(\phi_{j}-\phi_{i}, \lambda, T\right) v_{j}-\widetilde{G}\left(\phi_{j}-\phi_{i}, 0, T\right) v_{i}\right]
$$

where

$$
\widetilde{G}(\phi, \lambda, T)=\sum_{m \in \mathbb{Z}} G_{m}(\phi, T) \mathrm{e}^{-m \lambda}
$$

When $J(\tau)$ is given by an $\alpha$-function, the right-hand side of equation (2.20) reduces to a geometric series that can be summed explicitly (see $\S 2.4)$. One finds that $\widetilde{G}(\phi, \lambda, T)$ is an analytic function of $\lambda$ except for a pole at $\lambda=-\alpha T$. For more general choices of $J(t)$, it is no longer possible to explicitly sum the series in equation (2.20), and it is more convenient to work in the Fourier domain using the equivalent representation

$$
\widetilde{G}(\phi, \lambda, T)=\frac{1}{T} \sum_{m \in \mathbb{Z}}\left(i \omega_{m}+\lambda / T\right) \frac{\widetilde{J}\left(\omega_{m}-i \lambda / T\right)}{1+i \omega_{m}+\lambda / T}\left[\mathrm{e}^{\lambda}-\mathrm{e}^{-T}\right] \mathrm{e}^{2 \pi i m \phi+\lambda \phi}
$$

The Fourier series representation $(2.21)$ is convergent provided that $\widetilde{J}(\omega)$ decays faster than $1 / \omega$ as $\omega \rightarrow \pm \infty$, which is certainly holds true for the $\alpha$-function, see equation 
(2.13). This will also ensure that $\widetilde{G}(\phi, \lambda, T)$ is analytic in the right-hand complex $\lambda$-plane. A more rigorous derivation of the characteristic equation (2.14) in the particular case of a globally coupled network will be presented in $\S 4.2$.

One solution to equation (2.19) is $\lambda=0$ with $v_{i}=v$ for all $i$. This reflects the invariance of the dynamics with respect to uniform phase-shifts in the firing-times, $T_{i}^{n} \rightarrow T_{i}^{n}+v$. Thus the condition for linear stability of a phase-locked state is that all remaining solutions of equation (2.19) satisfy $\operatorname{Re} \lambda<0$. For sufficiently small $|g|$, solutions to equation (2.19) in the complex $\lambda$-plane will either be in a neighborhood of the real solution $\lambda=0$ or in a neighborhood of one of the poles of $\widetilde{G}(\phi, \lambda, T)$. Since the latter lie in the left-hand complex plane, the stability of the phase-locked solution will be determined by the eigenvalues in a neighborhood of the origin. Therefore, setting $\lambda=0$ on the right-hand side of (2.19) yields the equation

$$
\lambda\left(I_{i}-1\right) \delta_{i}=\frac{g}{T} \sum_{j=1}^{N} W_{i j} K^{\prime}\left(\phi_{j}-\phi_{i}, T\right)\left[\delta_{j}-\delta_{i}\right]+\mathcal{O}\left(g^{2}\right)
$$

where we have used the relation $T \widetilde{G}(\phi, 0, T)=K^{\prime}(\phi, T) \equiv \partial K(\phi, T) / \partial \phi$. Suppose for simplicity that $I_{i}=I$ for all $i=1, \ldots, N$. Then to $\mathcal{O}(g)$ the spectrum around the origin consists of the $N$ points $\lambda_{p}=(I-1) T_{0} \lambda_{p}^{(0)}, p=1, \ldots, N$, with $\lambda_{1}^{(0)}, \ldots, \lambda_{N}^{(0)}$ the eigenvalues of the $N \times N$ matrix $\widehat{\mathcal{J}}_{i j}(\Phi)=\mathcal{J}_{i j}(\Phi)-\delta_{i, j} \sum_{k=1}^{N} \mathcal{J}_{i k}(\Phi)$ where

$$
\mathcal{J}_{i j}(\Phi)=g W_{i j} K^{\prime}\left(\phi_{j}-\phi_{i}, T_{0}\right)
$$

and $T_{0}=\ln [I /(I-1)]$. If we choose $\lambda_{N}^{(0)}$ to be the zero eigenvalue then in the weak coupling limit the condition for stability reduces to $\operatorname{Re}\left(\lambda_{p}^{(0)}\right)<0$ for all $p=1, \ldots, N-1$. Note that such a stability condition could also be derived using a phase-reduced version of the IF model which, after a rescaling, takes the form $[32,8]$

$$
\frac{d \theta_{i}}{d t}=\frac{1}{T_{0}}+g \sum_{j=1}^{N} W_{i j} K\left(\theta_{j}-\theta_{i}, T_{0}\right)
$$

Phase-locked solutions of equation (2.24) are given by $d \theta_{i} / d t=\Omega$ where $\Omega$ is some $\mathcal{O}(g)$ correction to the natural frequency $1 / T_{0}$. Linearization about a phase-locked solution $\Phi$ generates the Jacobian $\widehat{\mathcal{J}}(\Phi)$.

2.3. Weak and strong instabilities. It is clear that a phase-locked solution of the phase model (2.24) is independent of the size of the coupling $|g|$. Therefore, destabilization of a phase-locked solution can only occur when variation of some other parameter such as the inverse rise-time $\alpha$ results in one or more eigenvalues of the Jacobian $\widehat{\mathcal{J}}(\Phi)$ crossing into the right-half complex plane. Equation (2.22) shows that a corresponding instability occurs in the weakly coupled IF model, and we shall refer to this as a weak instability. On the other hand, we shall define a strong instability as one in which a phase-locked solution $\Phi$ of the IF model is stable in the weak coupling regime but becomes unstable as $|g|$ is increased. A strong instability is signalled by one or more solutions of the full characteristic equation (2.19) crossing over into the righthalf complex $\lambda$-plane. If a single real eigenvalue $\lambda$ crosses the origin then the solution $\Phi$ will destabilize via a static bifurcation of the firing times. The bifurcating solutions will simply correspond to new phase-locked states and the oscillators will remain 1:1 frequency-locked. On the other hand, if a pair of complex conjugate eigenvalues 
crosses the imaginary axis at $\lambda= \pm i \omega$ then $\Phi$ will destabilize via a Hopf bifurcation in the firing times leading to the breakdown of 1:1 frequency-locking. In the special case $\omega=\pi$ this reduces to a period-doubling bifurcation. (A Hopf bifurcation in the firing times can also occur in the weak coupling regime but this does not lead to significant deviations from 1:1 frequency locking, see $\S 4.3)$.

2.4. Desynchronization via a discrete Hopf bifurcation. For simplicity, let us focus on the stability of the synchronous state for which $\phi_{i}=\phi$ for all $i=$ $1, \ldots, N$ and some arbitrary phase $\phi$. We also impose the homogeneity conditions $\sum_{j=1}^{N} W_{i j}=\Gamma, i=1, \ldots, N$ and $I_{i}=I \equiv I_{0}+\Delta I$ with

$$
\Delta I\left[1-\mathrm{e}^{-T_{0}}\right]+g K\left(0, T_{0}\right) \Gamma=0, \quad i=1, \ldots, N
$$

for some fixed $I_{0}>1$ and $T_{0}=\ln \left[I_{0} /\left(I_{0}-1\right)\right]$. The synchronous state then exists as a solution to equations (2.5) with collective period $T_{0}$. In order to determine the stability of the synchronous state, set $\phi_{i}=\phi$ in equation (2.19) and impose (2.25). Taking $\mathbf{v}=\left(v_{1}, \ldots, v_{N}\right)$ in equation (2.19) to be one of the eigenvectors of the weight matrix $\mathbf{W}$ with corresponding eigenvalue $\nu$ leads to the result

$$
A\left(\mathrm{e}^{\lambda}-1\right)=g\left[\widetilde{G}\left(0, \lambda, T_{0}\right) \nu-\widetilde{G}\left(0,0, T_{0}\right) \Gamma\right]
$$

where $A=I-1+g \Gamma J\left(0, T_{0}\right)$. (Note that equation (2.26) can be derived more formally using the method of $z$-transforms along similar lines to $\S 4.2)$. We explicitly evaluate $\widetilde{G}(0, \lambda, T)$ using equations (2.18) and (2.20). First,

$$
\begin{gathered}
G_{m}(0, T)=F_{0}(T) \mathrm{e}^{-m \alpha T}-m F_{1}(T) \mathrm{e}^{-m \alpha T} \\
F_{0}(T)=\frac{\alpha^{2} \mathrm{e}^{-T}}{(1-\alpha)^{2}}\left[\left(1-\alpha T+\alpha^{2} T\right) \mathrm{e}^{(1-\alpha) T}-1\right] \\
F_{1}(T)=\frac{T \alpha^{3} \mathrm{e}^{-T}}{1-\alpha}\left[\mathrm{e}^{(1-\alpha) T}-1\right]
\end{gathered}
$$

Substituting (2.27) into (2.20) and summing the resulting geometric series shows that

$$
\widetilde{G}(0, \lambda, T)=\frac{F_{0}(T)}{1-\mathrm{e}^{-\alpha T-\lambda}}-\frac{F_{1}(T) \mathrm{e}^{-\alpha T-\lambda}}{\left(1-\mathrm{e}^{-\alpha T-\lambda}\right)^{2}}
$$

It is clear from equation (2.30) that $\widetilde{G}(0, \lambda, T)$ has a pole at $\lambda=-\alpha T, n \in \mathbb{Z}$.

From equation (2.22) we have the following result concerning the stability of the synchronous state in the weak coupling regime. For sufficiently small coupling strength $|g|$, the synchronous state is linearly stable if and only if

$$
g K^{\prime}\left(0, T_{0}\right)\left[\operatorname{Re} \nu_{p}-\Gamma\right]<0, \quad p=1, \ldots, N-1
$$

where $\nu_{p}, p=1, \ldots, N$ are the eigenvalues of the matrix $\mathbf{W}$. Since $\sum_{j=1}^{N} W_{i j}=\Gamma$, it follows that one of the eigenvalues, $\nu_{N}$ say, satisfies $\nu_{N}=\Gamma$ with its eigenvector $\mathbf{v}_{N}=(1, \ldots, 1)$ corresponding to a uniform phase-shift. Evaluating $K(\phi, T)$ using equation (2.10), one can easily establish that $K^{\prime}(0, T)<0$ for all finite $\alpha$ and $T$. Thus the synchronous state is stable for sufficiently small $|g|$ provided that $g\left[\operatorname{Re} \nu_{p}-\Gamma\right]>0$ 
for all $p=1, \ldots, N-1$. Assuming the stability of the synchronous state for weak coupling, we can now consider what happens as the coupling strength $|g|$ is increased. It is simple to establish that the synchronous state cannot destabilize via a static bifurcation. However, it is possible for the synchronous state to undergo a discrete Hopf bifurcation in the firing times leading to a state with periodic or quasiperiodic firing patterns. In order to derive conditions for a Hopf bifurcation, set $\lambda=i \omega, \omega \neq 0$, and $\nu=\nu_{R}+i \nu_{I}$ for $\nu_{R}, \nu_{I} \in \mathbb{R}$ in equation (2.26). Equating real and imaginary parts then leads to the pair of equations

$$
\begin{aligned}
{[\cos (\omega)-1] A } & =g\left[\widetilde{C}(\omega) \nu_{R}+\widetilde{S}(\omega) \nu_{I}\right]-g \Gamma \widetilde{C}(0) \\
\sin (\omega) A & =g\left[\widetilde{C}(\omega) \nu_{I}-\widetilde{S}(\omega) \nu_{R}\right]
\end{aligned}
$$

where $\widetilde{C}(\omega)=\operatorname{Re} \widetilde{G}\left(0, i \omega, T_{0}\right), \widetilde{S}(\omega)=-\operatorname{Im} \widetilde{G}\left(0, i \omega, T_{0}\right)$. From equation (2.30) we derive the following expressions for $\widetilde{C}(\omega)$ and $\widetilde{S}(\omega)$ :

$$
\begin{aligned}
& \widetilde{C}(\omega)=a(\omega) C(\omega)-b(\omega)\left(\cos (\omega)\left[C(\omega)^{2}-S(\omega)^{2}\right]-2 \sin (\omega) C(\omega) S(\omega)\right) \\
& \widetilde{S}(\omega)=a(\omega) S(\omega)-b(\omega)\left(\sin (\omega)\left[C(\omega)^{2}-S(\omega)^{2}\right]+2 \cos (\omega) C(\omega) S(\omega)\right)
\end{aligned}
$$

where

$$
a(\omega)=\frac{F_{0}\left(T_{0}\right)}{C(\omega)^{2}+S(\omega)^{2}}, \quad b(\omega)=\frac{F_{1}\left(T_{0}\right) \mathrm{e}^{-\alpha T_{0}}}{\left[C(\omega)^{2}+S(\omega)^{2}\right]^{2}}
$$

and $C(\omega)=1-\mathrm{e}^{-\alpha T_{0}} \cos (\omega), S(\omega)=\mathrm{e}^{-\alpha T_{0}} \sin (\omega)$. The point at which the synchronous state becomes unstable is then given by the smallest value of the coupling $|g|=g_{c}$ for which a real non-zero solution $\omega$ of equation (2.32) exists.

It is important to note that linear stability analysis is not sufficient to determine (a) whether the Hopf bifurcation is supercritical or subcritical, nor (b) the nature of any stable states beyond the bifurcation point. In $\S 4.4$ and $\S 5$ we present numerical examples of strong instabilities in IF networks where desynchronization occurs via a Hopf bifurcation in the firing times. (Additional examples are given in Refs. [5, 6]). In all of these cases the bifurcation is found to be subcritical, that is, desynchronization involves a jump to a co-existing stable state typically characterized by inhomogeneous and (quasi)periodic variations of the inter-spike intervals (ISIs)

$$
\Delta_{k}^{n}=T_{k}^{n+1}-T_{k}^{n}
$$

on attracting invariant circles. These states can support a variety of complex dynamics including oscillator death ( $\$ 4.4$ ), bursting ( $\$ 5$ and Ref. [5]) and pattern formation [6]. A further result is that in the case of sufficiently slow synaptic interactions the resulting spatio-temporal variation of the ISIs is compatible with the behavior of a corresponding rate or analog model in which the outputs of the neurons are taken to be short-term firing rates. The relationship between strong instabilities of IF networks and analog networks is developed analytically in $\S 3$.

3. Strong instabilities and rate models. In order to establish a connection between strong instabilities in an IF network and a corresponding rate or analog network, we first rewrite the nonlinear firing time map (2.15) in a more suggestive form. Let

$$
G_{j}(t)=\int_{-\infty}^{0} \mathrm{e}^{s} \sum_{m \in \mathbb{Z}} J\left(s+t-T_{j}^{m}\right) d s
$$


Then equation (2.15) becomes

$$
\mathrm{e}^{T_{i}^{n+1}}\left[I-1+g \sum_{j=1}^{N} W_{i j} G_{j}\left(T_{i}^{n+1}\right)\right]=\mathrm{e}^{T_{i}^{n}}\left[I+g \sum_{j=1}^{N} W_{i j} G_{j}\left(T_{i}^{n}\right)\right]
$$

We now assume that the delay distribution $J(t)$ is a slowly decaying function of time, which in the case of synaptic delays (2.4) means that $\alpha$ is sufficiently small (slow synaptic interactions). We can then make the following approximation $G_{j}\left(T_{j}^{n+1}\right) \approx$ $G_{j}\left(T_{j}^{n}\right)$ for all $j, n$. This allows us to solve equation (3.2) as

$$
T_{i}^{n+1}-T_{i}^{n}=\ln \left[\frac{X_{i}\left(T_{i}^{n}\right)+I}{X_{i}\left(T_{i}^{n}\right)+I-1}\right]
$$

where

$$
X_{i}(t)=g \sum_{j=1}^{N} W_{i j} G_{j}(t)
$$

The next step is to replace the discrete sum in equation (3.1) by an integral (under the assumption of slow synapses):

$$
\sum_{m \in \mathbb{Z}} J\left(t-T_{j}^{m}\right) \approx \int_{-\infty}^{\infty} J(t-\tau) \frac{d \tau}{\Delta_{j}(\tau)}
$$

where $T_{j}^{m+1}-T_{j}^{m} \approx \Delta_{j}(\tau)$ for some smoothly varying function of time $\Delta_{j}(\tau)$. Substituting equations (3.1) and (3.5) into (3.4) then gives

$$
X_{i}(t)=g \sum_{j=1}^{N} W_{i j} \int_{-\infty}^{\infty} \widehat{J}(t-\tau) \frac{d \tau}{\Delta_{j}(\tau)}
$$

where $\widehat{J}(t)=\int_{-\infty}^{0} \mathrm{e}^{s} J(s+t) d s$. Finally, from equation (3.3) we have that $1 / \Delta_{j}(t)=$ $f\left(I+X_{j}(t)\right)$ where $f$ is the steady-state firing rate function

$$
f(X)=\left\{\ln \left[\frac{X}{X-1}\right]\right\}^{-1} \Theta(X-1)
$$

Thus we obtain the closed set of integral equations

$$
X_{i}(t)=g \sum_{j=1}^{N} W_{i j} \int_{0}^{\infty} J(\tau) f\left(X_{j}(t-\tau)\right) d \tau
$$

for the analog version of the IF model. (In equation (3.8) we have replaced $\widehat{J}$ by $J$. This does not lead to significant differences, particularly in the case of slow synapses). Equation (3.8) is a version of the Wilson-Cowan equation [35], although here it is obtained by a form of time-averaging under the assumption of slow synapses rather than by population averaging.

A more heuristic way of obtaining equation (3.8) is to suppose that the synaptic interactions are sufficiently slow so the total synaptic current into a neural oscillator is described by a slowly varying function of time $t$. If the neuronal dynamics is 
fast relative to $\alpha^{-1}$ then the actual firing rate $E_{i}(t)$ of a neuron will quickly relax to approximately its steady state value. We can then replace equation (2.3) by the approximation $E_{i}(t)=f\left(I_{i}+X_{i}(t)\right)$ with $f$ given by equation $(3.7)$ and $X_{i}(t)$ satisfying the integral equation (3.8). (For an additional discussion of how rate models can be derived from IF models see Ref.[1, 12]).

A common starting point for the analysis of analog models such as (3.8) is to consider conditions under which destabilization of a homogeneous low activity state occurs leading to the formation of a state with inhomogeneous and/or time-dependent firing rates [13]. To simplify our analysis, we shall impose the following condition on the external bias $I_{i}$ :

$$
I=I_{i}+g f(I) \sum_{j=1}^{N} W_{i j}
$$

for some fixed $I>1$. Then for sufficiently weak coupling, $|g| \ll 1$, the analog model has a single stable fixed point given by $X_{i}=g f(I) \sum_{j} W_{i j}$ such that the firing-rates are kept at the value $f(I)$. A state with homogeneous firing rates is analogous to a phase-locked state of an IF network, where all neurons have the same time-independent ISI. Note, however, that any phase information has been lost in the reduction procedure from the IF to the rate model. Suppose that we now linearize equation (3.8) about the homogeneous state and substitute into the linearized equations a solution of the form $X_{k}(t)=\mathrm{e}^{\lambda t} \bar{X}_{k}$. This leads to the eigenvalue equation

$$
\frac{\lambda_{p}^{ \pm}}{\alpha}=-1 \pm \sqrt{g f^{\prime}(I) \nu_{p}}, \quad p=1, \ldots, N .
$$

where $\nu_{p}, p=1, \ldots, N$, are the eigenvalues of the weight matrix $\mathbf{W}$. The fixed point will be asymptotically stable if and only if $\operatorname{Re} \lambda_{p}<0$ for all $p$. As $|g|$ is increased from zero, an instability may occur in at least two distinct ways. If a single real eigenvalue $\lambda$ crosses the origin in the complex $\lambda$-plane then a static bifurcation can occur leading to the emergence of additional fixed point solutions which correspond to inhomogeneous firing rates. For example, if $g>0$ and $\mathbf{W}$ has real eigenvalues $\nu_{1}>\nu_{2}>\ldots>\nu_{N}$ with $\nu_{1}>0>\nu_{N}$ then a static bifurcation will occur at the critical coupling $g_{c}$ for which $\lambda_{1}^{+}=0$, that is, $1=g_{c} f^{\prime}(I) \nu_{1}$. Similarly, if $g<0$ then $1=g_{c} f^{\prime}(I) \nu_{N}$. On the other hand, if a pair of complex conjugate eigenvalues $\lambda=\lambda_{R} \pm i \lambda_{I}$ crosses the imaginary axis $\left(\lambda_{R}=0\right)$ from left to right in the complex plane then a Hopf bifurcation can occur leading to the formation of periodic solutions, that is, time-dependent firing rates.

In cases where the rate model bifurcates to a state with inhomogeneous timeindependent firing rates, we expect from the above analysis that (quasi)periodic variations in the ISIs of the IF model to be small relative to the mean ISI (at least for slow synapses). That is, $\left|\Delta_{i}^{m}-\bar{\Delta}_{i}\right| \ll \bar{\Delta}_{i}$ for all $i, m$, where

$$
\bar{\Delta}_{i}=\lim _{M \rightarrow \infty} \frac{1}{2 M+1} \sum_{m=-M}^{M} \Delta_{i}^{m}
$$

However, $\bar{\Delta}_{i}$ will vary with $i$ (see for example $\S 4.4$ ). On the other hand, in cases where the rate model destabilizes to a state with time-dependent firing rates, we expect the (quasi)periodic variations in the ISIs to be relatively large (see §5). Incidentally, if there exists a stable homogeneous state of an analog network in the weak coupling regime then we expect 1:1 frequency locking to approximately hold under weak instabilities of the corresponding IF network (see $\S 4.3$ ). 
4. Weak and strong instabilities in globally coupled networks. In this section we consider weak and strong instabilities in a globally coupled network of identical IF oscillators for which $I_{i}=I, W_{i i}=0$ and $W_{i j}=1 /(N-1)$ for all $j \neq i$, $i=1, \ldots, N$. We also use this example to address a number of important technical issues concerning the stability of phase-locked solutions that have been ignored in previous treatments, including a specification of the class of perturbations with respect to which local stability is defined.

4.1. Phase-locking and symmetries. In the case of a globally coupled network the phase-locking equations (2.5) become

$$
1=I\left[1-\mathrm{e}^{-T}\right]+\frac{g}{N-1} \sum_{k \neq j} K\left(\phi_{j}-\phi_{i}, T\right)
$$

As we have discussed elsewhere [10], group theoretic methods can be used to classify the various solutions to equation (4.1) along similar lines to the analysis of phase oscillators by Ashwin and Swift [2]. Equation (4.1) is invariant under the action of the symmetry group $\Gamma=\mathbf{S}_{N} \times \mathcal{S}^{1}$ where $\mathbf{S}_{N}$ is the group of permutations of $N$ objects and $\mathcal{S}^{1}$ represents a phase-shift symmetry corresponding to time-translation invariance. That is, if $\Phi=\left(\phi_{1}, \ldots, \phi_{N}\right)$ is a solution of equation (4.1) then so is $\sigma \Phi$ for all $\sigma \in \Gamma$. The symmetries of any particular solution $\Phi$ form a subgroup called the isotropy subgroup of $\Phi$ defined by $\Sigma_{\Phi}=\{\sigma \in \Gamma: \sigma \Phi=\Phi\}$. More generally, we say that $\Sigma$ is an isotropy subgroup of $\Gamma$ if $\Sigma=\Sigma_{\Phi}$ for some $\Phi \in \mathbb{T}^{N}$. (Isotropy subgroups are defined up to some conjugacy; a group $\Sigma$ is conjugate to a group $\widehat{\Sigma}$ if there exists $\sigma \in \Gamma$ such that $\left.\widehat{\Sigma}=\sigma^{-1} \Sigma \sigma\right)$. The fixed-point subspace of an isotropy subgroup $\Sigma$, denoted by $\operatorname{Fix}(\Sigma)$, is the set of points $\Phi \in \mathbb{T}^{N}$ that are invariant under the action of $\Sigma, \operatorname{Fix}(\Sigma)=\left\{\Phi \in \mathbb{T}^{N}: \sigma \Phi=\Phi \forall \sigma \in \Sigma\right\}$.

TABLE 4.1

Some special isotropy subgroups $\Sigma$ of $\Gamma=\mathbf{S}_{N} \times \mathcal{S}^{1}$.

\begin{tabular}{llll}
\hline Isotropy $\Sigma$ & $\operatorname{Fix}(\Sigma)$ & $\operatorname{dim} \operatorname{Fix}(\Sigma)$ & Multiplicity \\
\hline $\mathbf{S}_{N}$ & $(\phi, \ldots, \ldots, \phi)$ & 1 & 1 \\
$\mathbf{Z}_{N}$ & $(\phi, \phi+1 / N, \phi+2 / N, \ldots, \phi-1 / N)$ & 1 & $(n-1) !$ \\
$\left(\mathbf{S}_{k}\right)^{m} \times \mathbf{Z}_{m}$ & $(\phi, \ldots, \phi, \phi+k / N, \ldots, \phi-k / N)$ & 1 & $n ! / m(k !)$ \\
& & & $(n=m k)$ \\
$(\mathbf{S})_{k_{1}} \ldots \times(\mathbf{S})_{k_{l}}$ & $\left(\phi_{1}, \ldots, \phi_{1}, \phi_{2}, \ldots, \phi_{l}\right)$ & $l$ & $n ! /\left(k_{1} ! \ldots k_{l} !\right)$ \\
\hline
\end{tabular}

One can now adopt a strategy that restricts the search for solutions of equation (4.1) to those that are fixed points of a particular isotropy subgroup. If $\operatorname{dim}(\operatorname{Fix}(\Sigma))=$ $d$ then equation (4.1) reduces to $d$ independent equations. In the special case of socalled maximally symmetric solutions for which $d=1$, equation (4.1) reduces to a single equation for the collective period $T$. In other words, the underlying symmetry of the system guarantees the existence of maximally symmetric solutions, assuming that a self-consistent $T$ can be found. (This is an example of the equivariant branching lemma [19]). Variation of one or more parameters such as $I, g, \alpha$ can induce bifurcations from a maximally symmetric solution to a solution with a smaller isotropy subgroup such as cluster states [18]. Some of the isotropy subgroups of $\Gamma=\mathbf{S}_{N} \times \mathcal{S}^{1}$ and their fixed-point spaces for equation (4.1) are shown in table 1. Of particular interest are the synchronous or in-phase solution, $\phi_{j}=\phi$ for all $j=1, \ldots, N$, and the splay or rotating wave states $\phi_{j}=\phi \pm j / N$. Both of these are maximally symmetric 
solutions whose period $T$ can be obtained by substituting $\phi_{j}=-\gamma j / N$ into equation (4.1) with $\gamma= \pm 1$ corresponding to the splay states and $\gamma=0$ corresponding to the in-phase state:

$$
1=I\left[1-\mathrm{e}^{-T}\right]+\frac{g}{N-1} \sum_{k=1}^{N-1} \sum_{m \in \mathbb{Z}} \int_{0}^{T} \mathrm{e}^{t-T} J(t+(m+\gamma k / N) T) d t
$$

4.2. Stability of splay and in-phase states: finite $N$. As discussed in $\S 2$ the stability of phase-locked solutions can be analyzed by considering perturbations of the firing times. These perturbations evolve according to a nonlinear firing time map, which is in the form of a difference equation of infinite order. Conditions for the local asymptotic stability of a phase-locked solution can be expressed in terms of the solutions of the characteristic equation (2.19) obtained by linearizing this firing time map. However, certain care has to be taken when dealing with infinite order systems since there are subtle issues concerning (i) the class of perturbations for which the stability analysis is valid, and (ii) the time-ordering of the firing times. Therefore, we shall follow a more rigorous treatment of stability here based on the use of generalized $z$-transforms. We shall focus on the stability of the in-phase and splay states although the analysis can be extended to phase-locking in more general networks.

The analysis of $\S 2$ establishes that the local stability of a phase-locked state depends on the asymptotic behavior of the linearized firing time map described by equation (2.16). In the case of the in-phase $(\gamma=0)$ or splay-states $(\gamma= \pm 1)$ of a globally coupled network this becomes

$$
A\left[u_{j}^{n+1}-u_{j}^{n}\right]=\frac{g}{N-1} \sum_{k \neq j} \sum_{m \in \mathbb{Z}} G_{m}(\gamma(j-k) / N, T)\left[u_{k}^{n-m}-u_{j}^{n}\right]
$$

where

$$
A=I-1+\frac{g}{N-1} \sum_{k=1}^{N-1} J(\gamma k / N, T) .
$$

and $G_{m}$ is defined by equation (2.18). In order to determine the asymptotic behavior of equation (4.3), it is necessary to specify initial data for the linear system, that is, the class of perturbations with respect to which local stability is defined. Therefore, we introduce the sets

$$
\begin{aligned}
& \mathcal{I}_{+}=\{(k, n)|k=1, \ldots, N, n \in \mathbb{Z}| n+\gamma k / N>0\} \\
& \mathcal{I}_{-}=\{(k, n)|k=1, \ldots, N, n \in \mathbb{Z}| n+\gamma k / N \leq 0\}
\end{aligned}
$$

and consider bounded initial data of the form $u_{j}^{n}=\phi_{j}^{n}$ for all $(j, n) \in \mathcal{I}_{-}$where $\max \left\{\left|\phi_{j}^{n}\right|,(j, n) \in \mathcal{I}_{-}\right\}<\infty$. We then carry out the decomposition

$$
u_{j}^{n}=v_{j}^{n} \Theta(n+\gamma j / N)+\phi_{j}^{n} \bar{\Theta}(n+\gamma j / N)
$$

with $\bar{\Theta}(x)=1-\Theta(x)$. In other words, we treat all firing times occurring before $t=0$ as initial data and solve for the firing times occurring after $t=0$. Substitution of equation (4.6) into (4.3) gives (for $n+1+\gamma j / N>0$ )

$$
\begin{aligned}
A v_{j}^{n+1} & =A v_{j}^{n} \Theta(n+\gamma j / N)+\frac{g}{N-1} \sum_{k \neq j} \sum_{m \in \mathbb{Z}} G_{m}(\gamma(j-k) / N, T) \\
& \times\left[v_{k}^{n-m} \Theta(n-m+\gamma k / N)-v_{j}^{n} \Theta(n+\gamma j / N)\right]+\left[F^{\Phi}\right]_{j}^{n}
\end{aligned}
$$


Equation (4.7) is now treated as an inhomogeneous linear equation for $v_{j}^{n}$ with the dependence on initial data collected together as a single external input term $\left[F^{\Phi}\right]$ with

$$
\begin{aligned}
{\left[F^{\Phi}\right]_{j}^{n} } & =A \phi_{j}^{n} \bar{\Theta}(n+\gamma j / N)+\frac{g}{N-1} \sum_{k \neq j} \sum_{m \in \mathbb{Z}} G_{m}(\gamma(j-k) / N, T) \\
& \times\left[\phi_{k}^{n-m} \bar{\Theta}(n-m+\gamma k / N)-\phi_{j}^{n} \bar{\Theta}(n+\gamma j / N)\right]
\end{aligned}
$$

In order to solve the inhomogeneous equation (4.7), we introduce the generalized $z$-transform $\mathcal{F}(u)$,

$$
\mathcal{F}(u)(z, p)=\sum_{n \in \mathbb{Z}} \sum_{k=1}^{N} \mathrm{e}^{-i p k} z^{-(n+\gamma k / N)} \Theta(n+\gamma k / N) u_{k}^{n}
$$

where $p=2 \pi q / N, q=0,1, \ldots, N-1$. Assume that the solutions $u_{k}^{n}$ are exponentially bounded in the sense that $u_{k}^{n}<\kappa r^{n+\gamma k / N}$ for $n+\gamma k / N>0$ and constants $\kappa, r$. Note that for the splay state, the time-like variable in equation (4.9) scales as $z^{-(n+k / N)}$, which takes into account the ordering of the firing times, (cf. the analysis of convective instabilities). Applying the transform $\mathcal{F}$ to equation (4.7) then gives

$$
A[z-1] \widetilde{v}(z, p)=\frac{g}{N-1}[\widetilde{B}(z, p)-\widetilde{B}(1,0)] \widetilde{v}(z, p)+\widetilde{F}^{\Phi}(z, p)
$$

where $\widetilde{F}^{\Phi}=\mathcal{F}\left(F^{\Phi}\right)$ and

$$
\widetilde{B}(z, p)=\sum_{k=1}^{N-1} \sum_{m \in \mathbb{Z}} \mathrm{e}^{-i p k} z^{-(m+\gamma k / N)} G_{m}(\gamma k / N, T)
$$

After a rearrangement of equation (4.10) we obtain

$$
\widetilde{v}(z, p)=\widetilde{X}(z, p) \widetilde{F}^{\Phi}(z, p), \quad \widetilde{X}(z, p)=\frac{z}{\widetilde{H}(z, p)}
$$

with

$$
\widetilde{H}(z, p)=(z-1) A-g[\widetilde{B}(z, p)-\widetilde{B}(1,0)]
$$

It can now be established that the asymptotic behavior of $u_{j}^{n}$ is governed by the zeros of $\widetilde{H}(z, p)$. First, note that one solution to this equation is $z=1$ at $p=0$, which reflects invariance of the system under uniform phase-shifts $T_{j}^{n} \rightarrow T_{j}^{n}+\delta$ for all $j, n$. This case will be excluded by requiring that the initial data $\Phi$ involves non-uniform perturbations. If we define

$$
r_{0}=\sup \{|z| \mid \widetilde{H}(z, p)=0, p=0,2 \pi / N, \ldots, 2 \pi(N-1) / N, z \neq 1\}
$$

then we can introduce the inverse transform

$$
X_{k}^{n}=\mathcal{F}^{-1}(\widetilde{X})(z, p) \equiv \frac{1}{N} \sum_{p} \oint_{C} \frac{d z}{2 \pi i z} \mathrm{e}^{i p k} \frac{z^{n+\gamma k / N}}{\widetilde{H}(z, p)}
$$

where $C=\left\{z \in \mathbb{C}|| z \mid>\min \left\{r_{0}, 1\right\}\right\}$ and $n+\gamma k / N>0$. We also define the convolution $u * v$ of two exponentially bounded sequences $u_{k}^{n}, v_{k}^{n}$ by

$$
[u * v]_{k}^{n}=\sum_{m \in \mathbb{Z}} \sum_{j=1}^{N} u_{k-j}^{m} v_{j}^{n-m} \Theta(m+\gamma(k-j) / N) \Theta(n-m+\gamma k / N)
$$


such that $\mathcal{F}(u * v)=\mathcal{F}(u) \mathcal{F}(v)$. The convolution theorem for $\mathcal{F}$ may be used to invert equation (4.12) leading to the solution

$$
u_{k}^{n}=\left[X * F^{\Phi}\right]_{k}^{n}, \quad n+\gamma k / N>0
$$

For bounded non-uniform initial data $\phi_{k}^{n}$,

$$
\left|u_{k}^{n}\right|<K r^{n+\gamma k / N}, \quad r>r_{0}, \quad n+\gamma k / N>0
$$

Hence, $\lim _{n \rightarrow \infty} u_{k}^{n}=0$ provided that all but one of the zeros of $\widetilde{H}(z, p)$ lie inside the unit circle.

The above analysis can be summarized in the following theorem:

THEOREM 4.1. Define the splay and in-phase states to be (locally) stable if and only if for all $\epsilon>0$ there exists $\delta>0$ such that if $\max \left\{\left|u_{k}^{m}\right|,(k, m) \in \mathcal{I}_{-}\right\}<\delta$ then $\max \left\{\left|u_{k}^{m}\right|,(k, m) \in \mathcal{I}_{+}\right\}<\epsilon$, where $u_{k}^{m}=T_{k}^{m}-(m+\gamma k / N) T$. The solution is defined to be asymptotically stable if it is locally stable and there exists $\delta>0$ such that if $\max \left\{\left|u_{k}^{m}\right|,(k, m) \in \mathcal{D}_{-}\right\}<\delta$ then $\lim _{n \rightarrow \infty} \max \left\{\left|u_{k}^{n}\right|\right\}=0$. (We exclude uniform initial data with respect to which a solution is always neutrally stable). We then have the following stability result: the splay state is (locally) asymptotically stable if the solutions of the characteristic equation

$$
(z-1) A=\frac{g}{N-1}[\widetilde{B}(z, p)-\widetilde{B}(1,0)]
$$

satisfy $|z|<1$ for all $p \in \mathbb{R}$ (except for one solution $z=1$ at $p=0$ ).

It is simple to establish that the characteristic equation (4.16) is equivalent to (2.19) for the given network. That is, set $A_{i}=A, W_{i j}=1 /(N-1), j \neq i$, and $W_{i i}=0$ for all $i=1, \ldots, N$ in equation (2.19) and then consider solutions of the form $z=\mathrm{e}^{\lambda}, \phi_{j}=\gamma j / N$ and $v_{j}=\mathrm{e}^{i p j-\lambda \phi_{j}}$. Note from equations (2.18), (2.20) and (4.11) that $\widetilde{B}\left(\mathrm{e}^{\lambda}, 0\right)=\widetilde{G}(0, \lambda, T)$ for a given collective period $T$. It is also possible to extend our stability analysis based on $z$-transforms to general networks and their phase-locked solutions using an appropriate diagonalization of the underlying matrix structure of the system.

4.3. Weak instability leading to partial synchronization. Recently, it has been found that in the case of excitatory coupling $(g>0)$, the splay state of a globally coupled IF network is stable for small $\alpha$ (slow synapses) but undergoes a Hopf bifurcation at some critical $\alpha_{c}$ [31]. Beyond the bifurcation point the neurons are no longer phase-locked and their inter-spike intervals (ISIs) vary either periodically or quasiperiodically on closed orbits. It was also observed that as one moves away from the bifurcation point $\left(\alpha>\alpha_{c}\right)$ the degree of coherence or synchrony of the network increases. Elsewhere we have established that this picture is considerably more subtle due to the occurrence of a global heteroclinic bifurcation in the firing times, which is a natural consequence of the underlying permutation symmetry of the network [8]. That is, for sufficiently large values of $\alpha$ one finds that the quasiperiodic orbit has been destroyed in a global heteroclinic bifurcation due to a collision with invariant sub-manifolds of the state-space. Coincident with this global bifurcation is a saddlenode bifurcation that creates pairs of stable/unstable cluster states. Beyond the bifurcation point the system is in a periodic state close to synchrony (corresponding to one of the newly created stable solutions). This saddle-node/heteroclinic bifurcation is analogous to the so-called transcritical/homoclinic bifurcation previously investigated for smoothly coupled phase oscillators with $\mathbf{S}_{N}$ symmetry (see [27]). 


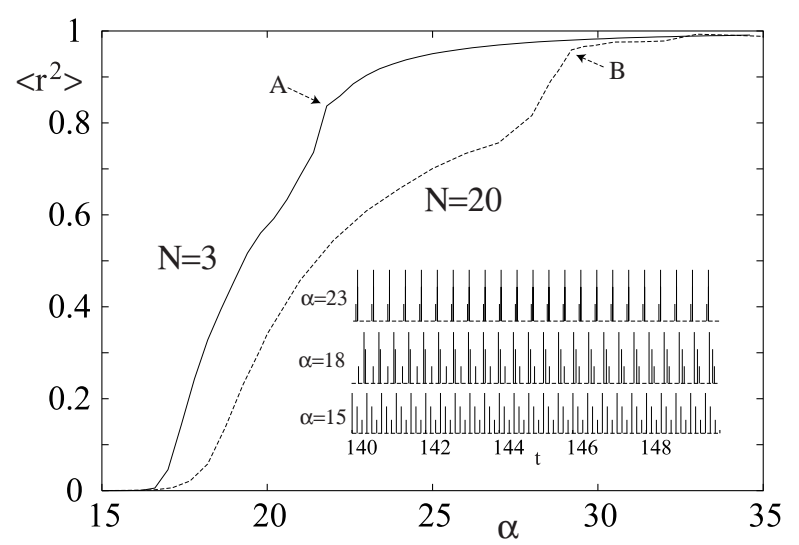

FIG. 4.1. Variation of the measure of synchrony $\left\langle r^{2}\right\rangle$ as a function of $\alpha$ for $N=3$ and $N=20$ with $g=0.4$, and $I=2$. Points $A$ and $B$ indicate where the dynamics has changed from a quasiperiodic to a periodic state due to a global heteroclinic bifurcation. Sample spike trains for $N=3$ are also shown with different neurons distinguished by the height of their spikes.

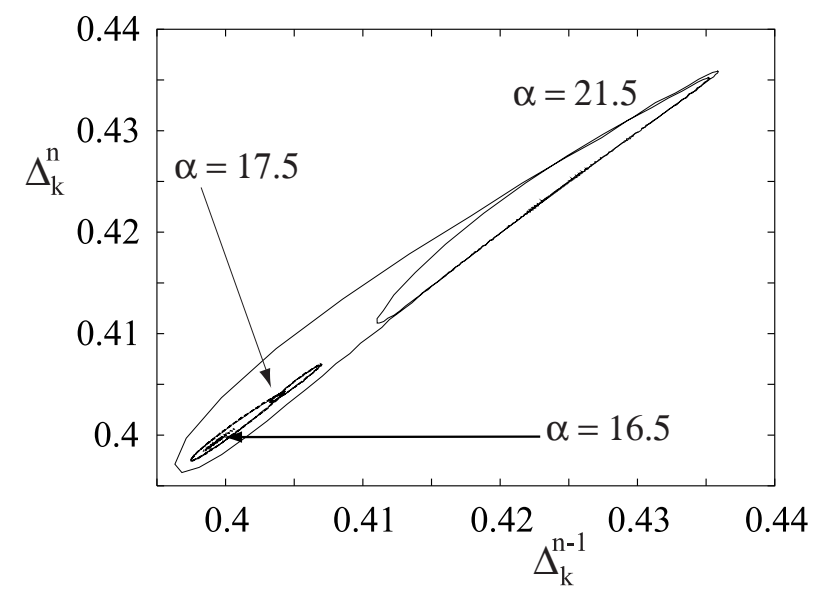

FIG. 4.2. Quasiperiodic motion of the ISIs on attracting invariant manifolds for $N=3$ with $g=0.4$ and $I=2$. This is an example of a weak instability in the firing times. Note that the temporal variation of the ISIs is relatively small in comparison to the mean ISI. (This should be contrasted with the strong instability shown in figure 5.5). For $\alpha<16$ the splay state is stable and the map of the ISIs has a fixed point at around 0.4. For $\alpha>22$ the two-in-phase state is stable, where two of the three oscillators fire in synchrony.

We shall illustrate the above instabilities by considering how the degree of synchrony in the network varies with $\alpha$. To quantify the degree of synchrony we use the firing time measure described in [28]. Firstly, we introduce a set of phases, $\phi_{k}(j, m)$, associated with the firing times of the $j$ th oscillator:

$$
\phi_{k}(j, m)=\frac{T_{k}(j, m)-T_{j}^{m}}{T_{j}^{m+1}-T_{j}^{m}}, \quad T_{j}^{m} \leq T_{k}(j, m)<T_{j}^{m+1}
$$

where the $T_{k}(j, m)$ represent the set of firing times of neurons $i \neq j$ that occur on the interval $\left[T_{j}^{m}, T_{j}^{m+1}\right)$. The number of such events will be denoted by $A(j, m)$ 
with $1 \leq k \leq A(j, m)$. For a set of phases $\Phi(j, m)=\left(\phi_{1}(j, m), \ldots \phi_{n}(j, m)\right)$ with $n=A(j, m)$ and fixed $(j, m)$ we introduce the order parameter

$$
r^{2}(\Phi)=\frac{1}{n^{2}} \sum_{k, l=1}^{n} \cos 2 \pi\left(\phi_{k}-\phi_{l}\right) .
$$

An overall measure of synchrony, $\left\langle r^{2}\right\rangle$, is defined by averaging $r^{2}(\Phi)$ over all oscillators and firing events in some time window. In Fig. 4.1, we show the variation of $\left\langle r^{2}\right\rangle$ for $N=3$ and $N=20$. For relatively small $\alpha$ the network is in a stable asynchronous state for which $\left\langle r^{2}\right\rangle=0$. At $\alpha=\alpha_{c}(N)$ there occurs a Hopf bifurcation to a partially synchronized state with $\left\langle r^{2}\right\rangle$ a smoothly increasing function of $\alpha$ until the network undergoes a global bifurcation to a nearly synchronous state with $\left\langle r^{2}\right\rangle \approx 1$. Note that in the mean-field limit (see also $\S 4.4$ ), the corresponding measure of synchrony in the thermodynamic limit does not appear to exhibit any effects due to a global bifurcation [31].

Within the context of the theory of spike train transitions presented in this paper, the destabilization of the splay state described above is an example of a weak instability since it can occur in the weak coupling regime. Indeed, a similar sequence of state transitions, namely a Hopf bifurcation from a splay state followed by a global bifurcation to a cluster state, are also observed in the corresponding phase model (2.24), see Ref. [8]. Although there are significant changes in the degree of synchrony, as highlighted in figure 4.1, the spatio-temporal variations in the ISIs characterized by the quasiperiodic orbits are relatively small [31, 8], which implies that 1:1 frequency locking still approximately holds. This is illustrated in figure 4.2 where we plot $\left(\Delta_{k}^{n}, \Delta_{k}^{n-1}\right)$ as a function of $n$ with $\Delta_{k}^{n} \equiv T_{k}^{n+1}-T_{k}^{n}$ the $n$th ISI of the $k$ th oscillator. In fact almost 1:1 frequency locking is expected to persist into the strong coupling regime. For using equation (3.10) is is easy to show that in the case of a globally coupled excitatory analog network, a homogeneous state will bifurcate to another homogeneous state as the coupling is increased (see §4.4).

4.4. Strong instability leading to oscillator death. Having discussed weak instabilities in a globally coupled excitatory network, we now consider strong instabilities in a corresponding inhibitory network for which $g<0$. This type of architecture has been used, for example, to model the reticular thalamic nucleus (RTN) [33, 18]. Note, however, that in the biophysical model of RTN developed by Wang and Rinzel [33], neural oscillations are sustained by post-inhibitory rebound rather than by an external bias as in our simple IF model. We shall show that for slow synapses desynchronization via a Hopf bifurcation in the firing times occurs, leading to oscillator death in the strong coupling regime, that is, certain cells suppress the activity of others. (See also the recent study of mutually inhibitory Hodgkin-Huxley neurons by White et al [34]). The occurrence of oscillator death is consistent with the behavior found in the rate model described by equation (3.8). For the weight matrix $\mathbf{W}$ has a non-degenerate eigenvalue $\nu_{+}=1$ with corresponding eigenvector $(1,1, \ldots, 1)$ and an $(N-1)$-fold degenerate eigenvalue $\nu_{-}=-1 /(N-1)$. It follows from equation $(3.10)$ that a homogeneous state of the rate model will destabilize at the critical coupling $|g|=g_{c}$ where $1=\sqrt{g_{c} f^{\prime}(I) /(N-1)}$. Moreover, it can be established that this corresponds to a subcritical bifurcation in which there are co-existent stable stationary states made up of active and inactive clusters. (An excitatory network would destabilize due to activation of the uniform mode $(1,1, \ldots, 1)$ leading to the formation of additional homogeneous states). 


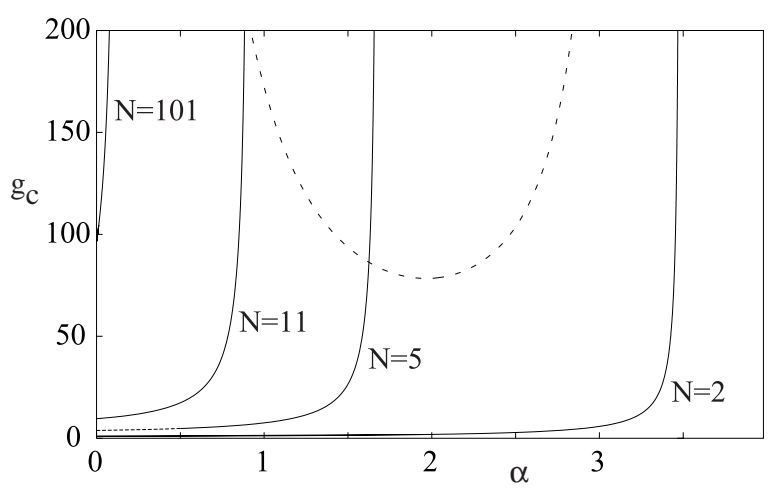

FIG. 4.3. Plot of critical coupling $g_{c}$ (solid curves) as a function of $\alpha$ for various network sizes $N$ and $T_{0}=\ln 2$. The critical inverse rise-time $\alpha_{0}(N)$ is seen to be a decreasing function of $N$ with $\alpha_{0}(N) \rightarrow 0$ as $N \rightarrow \infty$.

We shall analyze the stability of the synchronous state using the results of $\S 2.4$. First, set $I=I_{0}+\Delta$ such that equation (2.25) is satisfied (with $\Gamma=1$ ). The collective period of the synchronous solution is then fixed at $T_{0}$. Equation $(2.31)$ implies that the synchronous state is stable in the weak coupling regime since $\nu_{-}<\Gamma$ and $K^{\prime}\left(0, T_{0}\right)<0$ for $J(t)$ given by equation (2.4). We now investigate the stability of the synchronous state as $|g|$ is increased by solving equation (2.32). In Fig. 4.3 we plot the solutions $|g|=g_{c}$ of equation (2.32) as a function of the inverse rise-time $\alpha$ for $T_{0}=\ln 2$ and various network sizes $N$. The solid solution branches correspond to the eigenvalue $\nu_{-}$whereas the dashed branch corresponds to $\nu_{+}$and is $N$-independent. For small $N$ the solid branch determines the critical coupling $|g|=g_{c}(\alpha)$ for a Hopf instability. An important result that emerges from figure 4.3 is that there exists a critical value $\alpha_{0}(N)$ of the inverse rise-time beyond which a Hopf bifurcation cannot occur. That is, if $\alpha>\alpha_{0}(N)$ then the synchronous state remains stable for arbitrarily large inhibitory coupling. On the other hand, for $\alpha<\alpha_{0}(N)$ destabilization of the synchronous state occurs as $|g|$ crosses the solid branch from below. This signals the activation of an inhomogeneous state. Indeed, direct numerical simulation of the IF model shows that just after destabilization of the synchronous state $\left(|g|>g_{c}\right)$, the system jumps to an inhomogeneous state consisting of clusters of active and passive neurons. Moreover, the active neurons have approximately constant ISIs. Hence, for sufficiently slow synapses, the behavior of the IF model is compatible with that of the rate model. In the case of two inhibitory IF neurons $(N=2)$, the occurrence of oscillator death over a significant range of values of $\alpha$ shows that the stability criterion derived by Abbott et al [32] is necessary but not sufficient.

Interestingly, the critical value $\alpha_{0}(N)$ decreases with $N$, indicating the greater tendency for phase-locking to occur in large globally-coupled networks. This implies that for large networks, the neurons remain synchronized for arbitrarily large coupling even for slow synapses. There is one subtlety to be noted here. The dashed solution curve shown in Fig. 4.3 corresponds to excitation of the uniform mode $(1,1, \ldots, 1)$ and is independent of $N$. As $N$ increases it is crossed by the curve $g_{c}(\alpha)$ so that for a certain range of values of $\alpha$ it is possible for the synchronous state to destabilize due to excitation of the uniform mode. The neurons in the new state will still be synchronized but the spike trains will no longer have a simple periodic structure. 
The persistence of synchrony in large networks is consistent with the mode-locking theorem obtained by Gerstner et al (1996) in their analysis of the spike response model. We shall briefly discuss their result within the context of the IF model. For the given globally coupled network, the linearized map of the firing times, equation (4.3), becomes for the synchronous state

$$
A\left[u_{i}^{n+1}-u_{i}^{n}\right]=-g \sum_{m=-\infty}^{\infty} G_{m}(0, T)\left[\frac{1}{N-1} \sum_{j \neq i} u_{j}^{n-m}-u_{i}^{n}\right]
$$

The major assumption underlying the analysis of Gerstner et al [17] is that for large $N$ the mean perturbation $\left\langle u^{m}\right\rangle=\sum_{j \neq i} u_{j}^{m} /(N-1) \approx 0$ for all $m \geq 0$ say. Equation (4.19) then simplifies to the one-dimensional, first-order mapping

$$
u_{i}^{n+1}=\frac{I-1-g(I-1) K^{\prime}(0, T)}{I-1-g I K^{\prime}(0, T)} u_{i}^{n} \equiv k_{T} u_{i}^{n}
$$

The synchronous (coherent) state will be stable if and only if $\left|k_{T}\right|<1$. Equation (4.20) implies that the synchronous state is stable in the large $N$ limit provided that $K^{\prime}(0, T)<0$ (cf. equation $(2.31)$ ). This is the essence of the mode-locking theorem of Gerstner et al [17] applied to IF networks.

Finally, note that oscillator death has also been found in another class of homogeneous network, namely a one-dimensional network with long-range connections $W_{i j}=W(|i-j|)[6]$. The eigenvalues of $\mathbf{W}$ are $\nu(p)=2 \sum_{k=1}^{M} W(k) \cos (p k)$ for $p=0,2 \pi / N, \ldots, 2 \pi(N-1) / N$ with corresponding eigenmodes $v_{k}=\mathrm{e}^{i p k}$. If $W(k)$ is chosen to be a difference of Gaussians (representing short-range excitation and long-range inhibition), then solving equation (2.32) shows that a Hopf bifurcation in the firing times occurs due to excitation of an eigenmode $\mathrm{e}^{i p_{c} k}$ where $\nu\left(p_{c}\right)=\max _{p}\{\nu(p)\}, p_{c} \neq 0$. This strong instability leads to the formation of spatially periodic patterns consisting of alternating regions of activity and inactivity [6].

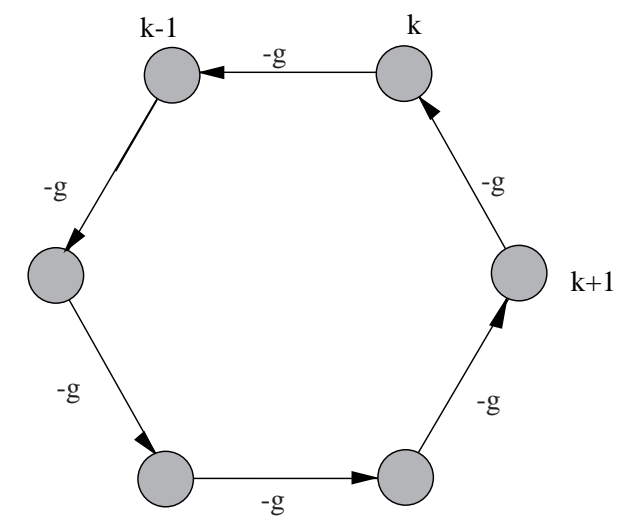

FIG. 5.1. A ring of IF oscillators with unidirectional inhibitory coupling

5. Bursting in a ring network. Consider a ring of identical IF neurons with unidirectional inhibitory coupling as shown in figure 5.1 with $g>0$. Let us first briefly look at a rate version of the ring network (see also [3]). For even numbered rings the 
eigenvalues of the weight matrix $\mathbf{W}$ are $\nu_{p}=\mathrm{e}^{2 \pi i p / N}, p=1, \ldots, N$. Since $\nu_{N}=1>$ Re $\nu_{p}$ for all $p \neq N$, it follows from equation (3.10) that the homogeneous state will undergo a static bifurcation at a critical coupling $g_{S}=1 / f^{\prime}(I)$ due to excitation of the eigenmode $(1,-1, \ldots,-1)$. This leads to the formation of an inhomogeneous state consisting of alternating active and inactive neurons around the ring. For odd numbered rings, the eigenvalues of the weight matrix $\mathbf{W}$ are $\nu_{p}=\mathrm{e}^{(2 p-1) \pi i / N}, p=$ $1, \ldots, N$. The homogeneous state now undergoes a subcritical Hopf bifurcation at a critical value of the coupling $g_{H}=\left[f^{\prime}(I) \cos (\pi / N)\right]^{-1}$ (see figure 5.2), which results in a time-dependent pattern of output activity as shown in figure 5.3. (Note that for the given network, if the firing rate function $f(X)$ were given by an odd function such as $\tanh (X)$ rather than equation (3.9), then the Hopf bifurcation would be supercritical $[3])$.

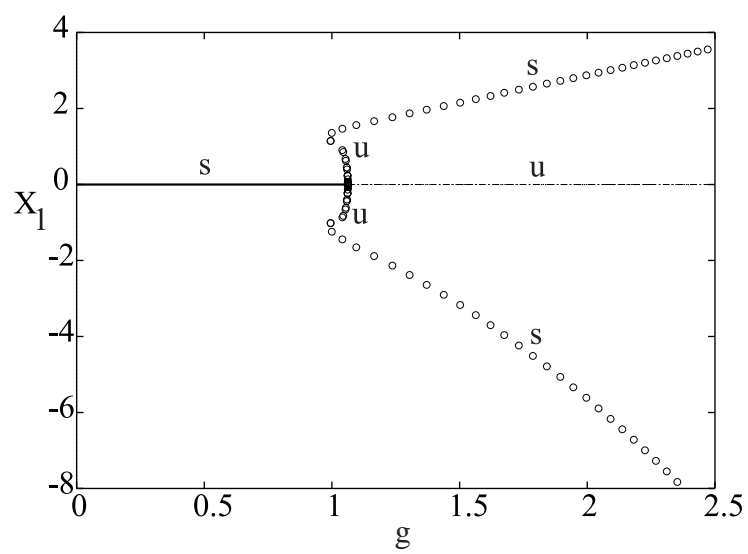

FIG. 5.2. An illustration of a Hopf bifurcation in the synaptic input $X_{1}$ for an analog model of 5 oscillators with uni-directional coupling. The amplitude of limit cycle oscillations is depicted with the use of open circles. Note that the bifurcation is a sub-critical one, leading to the creation of an unstable limit cycle and an unstable fixed point from the destruction of a stable fixed point. Parameters are $I=2$ and $\alpha=0.5$. $s$ (u) stands for stable (unstable) dynamics.

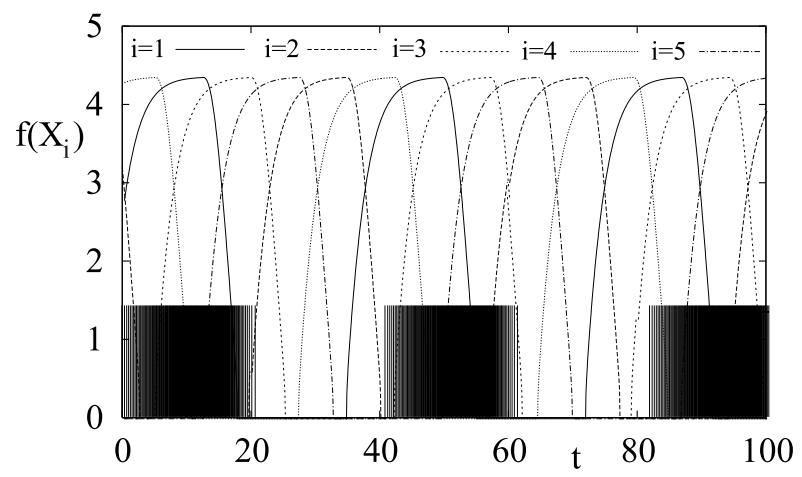

FIG. 5.3. Firing rates for a unidirectional ring of 5 analog neurons with $I=2, \alpha=0.5$ and $g=2$. The neuronal output of each of the neurons is identical up to a uniform phase-shift. Also shown is the spike train of one of the oscillators in an IF version of the ring network.

In the case of slow synapses, the above behavior is consistent with that found 
in the IF model. Since the analysis of even numbered rings is very similar to the case of oscillator death in a globally coupled inhibitory network (see $§ 4.4$ ), we shall focus on the case of odd $N$. The synchronous state is stable in the weak coupling regime. However, there exists a critical inverse rise-time $\alpha_{0}$ such that for $\alpha<\alpha_{0}$ the synchronous state destabilizes via a Hopf bifurcation at a critical coupling $g_{c}$, which is a solution to equation (4.8) with $\Gamma=-1, \nu_{R}=\cos (\pi / N)$ and $\nu_{I}= \pm \sin (\pi / N)$ (see figure 5.4). Direct numerical simulation shows that beyond the Hopf bifurcation point the oscillators exhibit periodic bursting patterns, which are identical up to a uniform phase-shift (see spike train in figure 5.3).

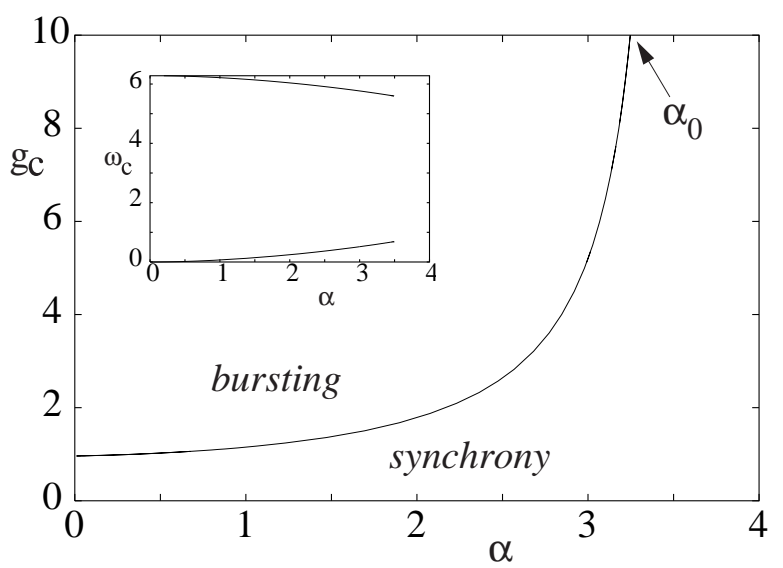

FIG. 5.4. Unidirectional inhibitory coupling in a ring of 5 identical IF oscillators. Self consistent solution of $\left(\omega_{c}, g_{c}\right)$ with $\nu_{R}=\cos (\pi / 5), \nu_{I}= \pm \sin (\pi / 5), I=2, \Gamma=-1$

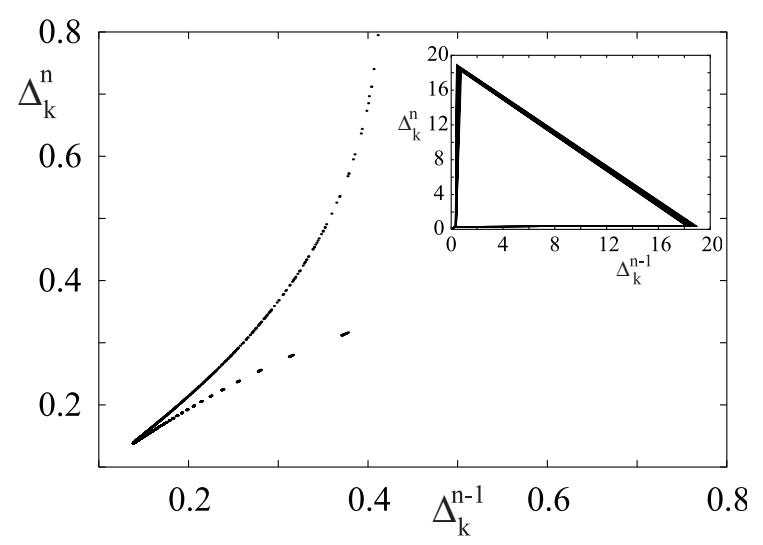

FIG. 5.5. A plot of the inter-spike intervals $\left(\Delta_{k}^{n-1}, \Delta_{k}^{n}\right)$ for a ring of 5 IF oscillators with unidirectional coupling, beyond the discrete Hopf bifurcation point of the linearized firing map, shows a projection of dynamics on an invariant circle. The main figure shows orbit points of the full nonlinear map of firing times within a burst (for all of the oscillators). In the inset we plot the full set of ISIs for all the oscillators connected by lines, highlighting the separation of the bursting patterns by relatively large periods of inactivity.

The occurrence of bursting can be understood in terms of mode-locking associated with periodic variations of the inter-spike intervals $\Delta_{k}^{n} \equiv T_{k}^{n+1}-T_{k}^{n}$ on attracting 
invariant circles. This is illustrated in figure 5.5, where we plot $\left(\Delta_{k}^{n-1}, \Delta_{k}^{n}\right)$ as a function of $n$ for a ring of $k=1, \ldots, 5$ oscillators. Each oscillator has a periodic solution of length $M$ such that $\Delta_{k}^{n+p M}=\Delta_{k}^{n}$ for all integers $p$. Moreover, for each $k$ there exists $l \in\{1, \ldots, M\}$ with $\Delta_{k}^{l} \gg \Delta_{k}^{n}$ for all $n \neq l$ so that the resulting spike train exhibits bursting with the inter-burst interval equal to $\Delta_{k}^{1}$ and the number of spikes per burst equal to $M$. It is interesting to note that although the data is taken for parameter values close to the bifurcation curves of figure 5.4, the, variation in the inter-spike intervals is large compared to $\sqrt{g-g_{c}}$. Moreover, the frequency $\omega$ of the variations in the inter-spike intervals differs significantly from the critical frequency $\omega_{c}$. This confirms that the Hopf bifurcation is subcritical rather than supercritical, as in the rate model (see figure 5.2). Finally, comparison of figure 5.5 with figure 4.2 shows that the strong coupling instability results in a significantly larger temporal variation in the ISIs than the weak coupling instability.

Note that the occurrence of bursting in a network of IF neurons is not specific to the particular ring architecture shown in figure 5.1. Indeed, we expect bursting to occur in any network for which the corresponding rate model destabilizes from a homogeneous state via a Hopf bifurcation in the firing rates. One well known example is that of an excitatory/inhibitory pair of neurons, which is studied along similar lines to the ring network in Ref. [5].

6. Conclusion. In this paper we have presented a general dynamical theory of spiking integrate-and-fire neurons that bridges the gap between weakly coupled phase oscillator models and strongly coupled firing rate models. The relative simplicity of the IF model allows precise analytical statements to be made. It is hoped that our work will provide insights into the complex types of behaviour expected in more biophysically detailed neural models.

\section{REFERENCES}

[1] D. J. Amit And M. V. Tsodyks, Quantitative study of attractor neural networks retrieving at low spike rates I: Substrate - spikes, rates and neuronal gain, Network, 2 (1991), pp. 259274 .

[2] P. Ashwin And J. W. Swift, The dynamics of $n$ weakly coupled identical oscillators, J. Nonlinear Sci., 2 (1992), pp. 69-108.

[3] A. ATIYA AND P. BALDI, Oscillations and synchronization in neural networks: an exploration of the labelling hypothesis, Int. J. Neural Syst., 1 (1989), pp. 103-124.

[4] P. C. Bressloff, Resonant-like synchronization and bursting in a model of pulse-coupled neurons with active dendrites, J. Comp. Neurosci. (in press), (1999).

[5] P. C. Bressloff and S. Coombes, Desynchronization, mode-locking and bursting in strongly coupled integrate-and-fire oscillators, Phys. Rev. Lett., (1998), pp. 2168-2171.

[6] - Spike train dynamics underlying pattern formation in networks of integrate-and-fire oscillators, Phys. Rev. Lett., (1998), pp. 2384-2387.

[7] — , Travelling waves in a chain of pulse-coupled oscillators, Phys. Rev. Lett., (1998), pp. $4815-4818$.

[8] - Symmetry and phase-locking in a ring of pulse-coupled oscillators with distributed delays, Physica D (in press), (1999).

[9] - Travelling waves in a chain of pulse-coupled integrate-and-fire oscillators with distributed delays, Physica D. (in press), (1999).

[10] P. C. Bressloff, S. Coombes, And B. D. Souza, Dynamics of a ring of pulse-coupled oscillators: a group theoretic approach, Phys. Rev. Lett., 79 (1997), pp. 2791-2794.

[11] S. CoOmbes And G. J. LORD, Desynchronisation of pulse-coupled integrate-and-fire neurons, Phys. Rev. E, 55 (1997), pp. 2104R-2107R.

[12] G. B. ERmentrout, Reduction of conductance-based models with slow synapses to neural nets, Neural Comput., 6 (1994), pp. 679-695. 
[13] — N _ Neural networks as spatio-temporal pattern-forming systems, Rep. Prog. Phys., 61 (1998), pp. 353-430.

[14] G. B. Ermentrout And N. Kopell, Frequency plateaus in a chain of weakly coupled oscillators, SIAM J. Math. Anal., 15 (1984), pp. 215-237.

[15] U. Ernst, K. Pawelzik, and T. Giesel, Synchronization induced by temporal delays in pulsecoupled oscillators, Phys. Rev. Lett., 74 (1995), pp. 1570-1573.

[16] W. Gerstner, Time structure of the activity in neural-network models, Phys. Rev. E, 51 (1995), pp. $738-758$.

17] W. Gerstner, J. L. van Hemmen, and J. D. Cowan, What matters in neuronal locking, Neural Comput., 94 (1996), pp. 1653-1676.

[18] D. Golomb and J. Rinzel, Clustering in globally coupled inhibitory neurons, Physica D, 72 (1994), pp. 259-282.

[19] M. Golubitsky, I. N. Stewart, and D. G. Schaeffer, Singularities and Groups in Bifurcation Theory, vol. 2 of Applied mathematical sciences;69, New York:Springer-Verlag, 1988.

[20] C. M. GraY, Synchronous oscillations in neuronal systems: mechanisms and functions, J. Comput. Neurosci., 1 (1994), pp. 11-38.

[21] D. Hansel, G. Mato, and C. Meunier, Synchrony in excitatory neural networks, Neural Comput., 7 (1995), pp. 307-337.

[22] A. L. Hodgkin And A. F. Huxley, A quantitative description of membrane current and its application to conduction and excitation in nerve, J. Physiol. (London), 117 (1952), pp. $500-544$.

[23] J. J. B. Jack, D. Noble, and R. W. Tsien, Electric Current Flow in Excitable Cells, Clarendon Press, Oxford, 1975.

[24] W. M. Kistler, R. Seitz, and J. L. van Hemmen, Modeling collective excitations in cortical tissue, Physica D, 114 (1998), pp. 273-295.

[25] N. Kopell, Chains of coupled oscillators, in The Handbook of Brain Theory and Neural Networks, M. A. Arbib, ed., MIT Press, 1995, pp. 178-183.

[26] R. E. Mirollo and S. H. Strogatz, Synchronisation of pulse-coupled biological oscillators, SIAM J. Appl. Maths, 50 (1990), pp. 1645-1662.

[27] G. P. K. P Ashwin AND J. W. SWIFT, Three identical oscillators with symmetric coupling, Nonlinearity, 3 (1990), pp. 585-601.

[28] P. F. Pinsky AND J. RinzeL, Synchrony measures for biological neural networks, Biol. Cybern., 73 (1995), pp. 129-137.

[29] R. H. Rand AND P. J. Holmes, Bifurcation of periodic motions in two weakly coupled van der pol oscillators, Int. J. Nonlinear Mech., 15 (1980), pp. 387-399.

[30] H. C. TuCKwell, Introduction to Theoretical Neurobiology, vol. I, Cambridge University Press, 1988.

[31] C. van VreeswiJk, Partial synchronization in populations of pulse-coupled oscillators, Phys. Rev. E., 54 (1996), pp. 5522-5537.

[32] C. van Vreeswijk, L. F. Авbott, and G. B. Ermentrout, When inhibition not excitation synchronizes neural firing, J. Comp. Neurosci., 1 (1994), pp. 313-321.

[33] X.-J. Wang AND J. Rinzel, Alternating and synchronous rhythms in reciprocally inhibitory model neurons, Neural Comput., 4 (1992), pp. 89-97.

[34] J. A. White, C. C. Chow, J. Ritt, C. Soto-Trevino, And N. Kopell, Synchronization and oscillatory dynamics in heterogeneous mutually inhibited neurons, J. Comput. Neurosci., 5 (1998), pp. $5-16$.

[35] H. R. WILSON AND J. D. COWAN, Excitatory and inhibitory interactions in localized populations of model neurons, J. Biophys., 12 (1972), pp. 1-24.

[36] M. A. Wilson And J. M. Bower, Cortical oscillations and temporal interactions in a computer simulation of piriform cortex, J. Neurophysiol, 67 (1992), pp. 981-995. 